\title{
Gene-expression profiling in breast cancer: bespoke cancer therapy or more fiction than science?
}

\author{
M. Barry $\cdot$ M. R. Kell
}

Received: 5 March 2010/ Accepted: 19 August 2010/Published online: 31 August 2010

(C) Royal Academy of Medicine in Ireland 2010

Breast cancer is the most common life threatening malignancy in women. Despite advances in molecular therapeutics, many patients with apparently early stage and curable disease still develop progressive and fatal systemic disease. Haffty et al. [1] demonstrated that despite conservative surgery and radiation followed by administration of chemotherapy in early-stage (T1 tumours) breast cancer patients, the distant metastasis-free survival rate was $71 \%$ at 5 years in patients with triple negative disease and $83 \%$ at 5 years in non-triple negative. These patients have potentially been under-staged by conventional staging, and therefore may not have received optimum therapy due to their misleading clinical disease profile. Conversely, patients receiving systemic therapy may not derive any additional benefit. Breast cancer is an intricate genetic milieu characterised by numerous molecular alterations that prevents standardising therapeutic strategies to all patients. Until recently, it has been difficult to accurately profile and predict the biological properties and clinical behaviour of breast tumours, however, with the development of DNA microarray technology, it is now possible to analyse the RNA expression of several thousands of genes simultaneously. The clinical application of this technology has the potential to signal a paradigm shift in patient selection for systemic therapy.

First, it provided a new molecular classification of breast cancer. This classification was proposed by Perou et al. [2], and subdivided breast cancers into four classes. Luminal-A cancers are ER-positive and mostly low grade. Luminal-B

M. Barry · M. R. Kell ( $\square)$

Eccles Breast Screening Unit,

Mater Misericordiae University Hospital,

Eccles St, Dublin 7, Ireland

e-mail: malcolm.kell@breastcheck.ie cancers are also ER-positive but tend to be high grade. Basal-like cancers are triple negative and Her-2-like cancers are Her-2 positive and usually ER negative. Currently, there are a number of validated commercially available multigene assays that identify expression of distinct sets of genes in breast cancer: Mammaprint (Agendia), Oncotype DX (Genomic Health), MapQuant and H/I (AvariaDX) (see Table 1 for comparison of assays). Mammaprint uses DNA microarray technology, whereas Oncotype DX and H/I use real time PCR based assays. These assays accurately predict the potential for disease recurrence and enhance patient selection for chemotherapeutic strategies.

Mammaprint uses a 70-gene predictor and is involved in the MINDACT (Microarray In Node negative Disease may Avoid ChemoTherapy) trial [3]. The objective is to administer chemotherapy to patients deemed at high risk of recurrence, and hormonal therapy only to low risk patients, as determined by the Mammaprint gene profile. Having recruited large numbers of patients who are stratified based on the clinical and pathological risks, it is hoped that this study will provide important information regarding the prognostic capabilities of the Mammaprint test and also validate its use in identifying women associated with improved survival. Previously, Buyse et al. [4] demonstrated that Mammaprint outperformed Adjuvant Online in predicting survival in an independent group of patients. Unfortunately, however, a major disadvantage of Mammaprint is that it requires fresh tissue for analysis, which may make its clinical integration difficult. The Oncotype DX and H/I assay, however, can use archival tissue (tissue from original biopsy) and may be more readily applied to the clinical setting. By analysing the expression of 21 known genes, Oncotype DX accurately determined the prognostic outcome in hormone receptor positive cancers post adjuvant tamoxifen in a retrospective cohort of 668 
Table 1 Comparison of commercially available genomic assays

\begin{tabular}{lllll}
\hline Variable & Oncotype DX & Mammaprint & Theros & MapQuant Dx \\
\hline Type of assay & 21-gene & 70-gene & 2-gene ratio & 97-gene \\
Tissue & Formalin-fixed, paraffin embedded & Fresh/frozen & Formalin-fixed, paraffin embedded & Fresh/frozen \\
Cost & US $\$ 3,500$ & US $\$ 3,500$ & n/a & n/a \\
Technique & Q-RT-PCR & DNA microarrays & Q-RT-PCR & DNA microarrays
\end{tabular}

$Q-R T-P C R$ quantitative reverse-transcriptase-polymerase chain reaction

patients [5]. This was also validated by Habel et al. [6], who demonstrated that Oncotype DX was an accurate predictive test for ER-positive, node negative breast cancer patients who did not receive chemotherapy but offered tamoxifen. Furthermore, TAI-LORx (trial assigning individualised options for treatment) aims to assess the role of Oncotype DX as an accurate predictor of chemotherapeutic response for ER-positive and node negative tumours [7].

Numerous studies have examined gene profiles that are associated with tumour response to certain chemotherapeutic agents in both the adjuvant setting (adriamycin/ cyclophosphamide, docetaxel) and the neoadjuvant setting (paclitaxel/fluorouracil/cyclophosphamide/doxorubicin)

[8-11]. In addition, recent studies have also suggested that deletion and amplification of the topoisomerase II alpha (TOP2A) gene is a poor prognostic indicator and is predictive of an enhanced response for HER2 positive tumours to anthracycline-containing than to non-anthracyclinecontaining regimens10. Also the CYP2D6 enzyme is crucial in the metabolism of tamoxifen to its active metabolite and potent antiestrogen endoxifen. A subset of patients with low or completely deficient levels of CYP2D6 cannot activate tamoxifen, and are therefore unable to benefit from its antitumor effects $[12,13]$. A commercially available assay has now been developed which can identify these CYP2D6 deficient patients, AmpliChip (Roche Diagnostics Inc., Indianapolis, IN, USA). It is hoped that these tamoxifen insensitive CYP2D6 deficient patients with newly diagnosed breast cancer, may now receive an alternative adjuvant hormonal therapeutic strategy rather than tamoxifen. Combined, these studies demonstrate the potential to identify genes which will determine the level of response to a given treatment regimen. However, it is difficult to predict how soon these evolving technologies will be integrated into clinical practice. However, this promising technology has been evolving over the last 5 years and yet despite endorsements from the Food and Drug Adminstration (FDA), the American Society of Clinical Oncology (ASCO) and the National Comprehensive Cancer Network (NCCN), there is a surprising lack of robust prospective data. Currently, there are only two prospective randomised trials comparing genomic testing and clinical factors. The results of the European
MINDACT Trial and the American TAI-LORx Trial are eagerly awaited to determine the future application of gene-expression technology in the clinical setting $[3,7]$. Interestingly, a prospective multi-centre study, which examined the effectiveness of Mammaprint when used in conjunction with clinical guidelines, demonstrated an alteration in adjuvant treatment in $26 \%$ of patients [14]. In addition, a retrospective analysis of the impact of OncotypeDX low recurrence score results on treatment decisions demonstrated a change in management strategy in $30 \%$ of patients [15]. These findings suggest that this expensive technology may alter patient care in approximately onethird of cases and the clinical impact in terms of survival benefit of this alteration has yet to be determined. Genetic profiling of patients and their tumours promises to accurately select patients with a high/low risk of recurrence and more importantly predict their response to certain chemotherapeutic agents. However, until additional randomized studies with large cohorts of patients with various tumour types and stages are completed, it remains as only a promise. The integration of this technology into the clinical setting is dependent on the completion of ongoing trials and until this data is available and appropriately validated, gene-expression profiling is best used as an adjunct to current clinical algorithms. This technology has and will continue to enhance our understanding of oncogenesis, however, it is currently unclear how these advances will ultimately impact on future breast cancer therapy and patient care.

\section{References}

1. Haffty BG, Yang Q, Reiss M et al (2006) Locoregional relapse and distant metastasis in conservatively managed triple negative early-stage breast cancer. J Clin Oncol 24((36)):5652-5657

2. Perou CM, Sorlie T, Eisen MB et al (2000) Molecular portraits of human breast tumours. Nature 406:747-752

3. Cardoso F, Van't Veer L, Rutgers E et al (2008) Clinical application of the 70-gene profile: the MINDACT trial. J Clin Oncol 26((5)):729-735

4. Buyse M, Loi S, van't Veer L et al (2006) Validation and clinical utility of a 70-gene prognostic signature for women with nodenegative breast cancer. J Natl Cancer Inst 98:1183-1192 
5. Paik S, Shak S, Tang G et al (2004) A multigene assay to predict recurrence of tamoxifen-treated, node negative breast cancer. N Engl J Med 351((27)):2817-2826

6. Habel LA, Shak S, Jacobs MK et al (2006) A population-based study of tumor gene expression and risk of breast cancer death among lymph node-negative patients. Breast Cancer Res 8(3):R25

7. Sparano JA, Paik S (2008) Development of the 21-gene assay and its application in clinical practice and clinical trials. J Clin Oncol 26(5):721-728

8. Sotiriou C, Powles TJ, Dowsett M et al (2002) Gene expression profiles derived from fine needle aspiration correlate with response to systemic chemotherapy in breast cancer. Breast Cancer Res 4(3):R3

9. Chang JC, Wooten EC, Tsimelzon A et al (2003) Gene expression profiling for the prediction of therapeutic response to docetaxel in patients with breast cancer. Lancet 362(9381):362-369

10. Modlich O, Prisack HB, Munnes M et al (2004) Immediate gene expression changes after the first course of neoadjuvant chemotherapy in patients with primary breast cancer disease. Clin Cancer Res 10(19):6418-6431

11. Pritchard KI, Messersmith H, Elavathil L et al (2008) HER-2 and topoisomerase II as predictors of response to chemotherapy. J Clin Oncol 26(5):736-744

12. Jin Y, Desta Z, Stearns V et al (2005) CYP2D6 genotype, antidepressant use, and tamoxifen metabolism during adjuvant breast cancer treatment. J Natl Cancer Inst 97:30-39

13. Goetz MP, Knox SK, Suman VJ et al (2007) The impact of cytochrome P450 2D6 metabolism in women receiving adjuvant tamoxifen. Breast Cancer Res Treat 101:113-121

14. Bueno-de-Mesquita JM, van Harten WH, Retel VP et al (2007) Use of 70-gene signature to predict prognosis of patients with node negative breast cancers: a prospective community based feasibility study (RASTER). Lancet Oncol 8:1079-1087

15. Liang H, Burfsky AM, Lembersky BB et al (2007) A retrospective analysis of the impact of OncotypeDX low recurrence score results on treatment decisions in a single academic breast cancer center. Breast Cancer Res Treat 106:105 